\section{Kompass \\ Autoimmun}

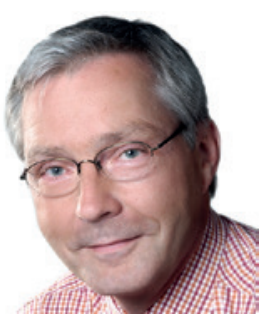

Michael Sticherling

Hautklinik, Universitätsklinikum Erlangen, Erlangen, Deutschland

Die Lunge gehört zu den lebenswichtigsten Organen des Menschen. Ein Totalausfall ist mit dem Leben nicht vereinbar, gleichzeitig ist ein Ersatz durch gesundes Gewebe mittels Organtransplantation oder technische Geräte bis heute problematisch. Angesichts beständiger Nutzung und in der Regel problemloser Funktion erfährt die Lunge meist wenig Aufmerksamkeit, bei Einschränkung ihrer Funktionsfähigkeit wird jedoch die Lebensqualität und Lebensfähigkeit der Betroffenen massiv beeinträchtigt. Gerade in der aktuellen Pandemie-Situation ist eine Lungenbeteiligung im Rahmen von COVID-19 hochaktuell und die Behandlung respiratorischer Beschwerden zentrale und mühsame Aufgabe bei der Betreuung der Betroffenen.

Luftnot gehört zu den eindringlichsten Angstzuständen des Menschen. Luftnot und Husten sind dabei unspezifische Sym-

\title{
Bei der Diagnostik von Autoimmunerkrankungen auch die Lunge einbeziehen
}

ptome, die einer zügigen und sauberen Abklärung bedürfen. Neben infektiösen und tumorösen Lungenerkrankungen sind insbesondere chronisch-entzündliche Veränderungen bedeutsam, die sich häufig langsam schleichend entwickeln und erst sehr verzögert diagnostiziert und eingeordnet werden. Gerade chronische Erkrankungen können aber zu einer nachhaltigen und permanenten Schädigung des Lungengewebes führen und müssen daher rechtzeitig erkannt und behandelt werden. Hier kann die Lunge als isoliertes Organen betroffen sein, aber auch als eines mehrerer Organe im Rahmen komplexerer Erkrankungen z.B. von Autoimmunerkrankungen. Daher ergeben sich häufig Überschneidungen mit anderen Spezialitäten wie der Rheumatologie, aber auch der Dermatologie. Das Lungenepithel in seinen verschiedenen anatomischen Organabschnitten, das interstitielle Lungengewebe wie auch das komplexe Blutgefäßsystem der Lunge können einzeln, häufig aber kombiniert und sequentiell betroffen sein mit jeweils spezifischer klinischer Symptomatik.

Im Rahmen der Reihe Kompass Autoimmun werden daher im aktuellen Heft verschiedene Aspekte von Lungenerkrankungen besprochen: die interstitielle Lungenerkrankung, die pulmonal arterielle Hypertonie, bösartige Lungenerkrankungen wie auch die Lungenbeteiligung im Rahmen rheumatologischer Erkrankungen wie den Systemvaskulitiden oder der Dermatomyositis. Wir wünschen Ihnen viel Spaß und Informationsgewinn beim Lesen Ihr

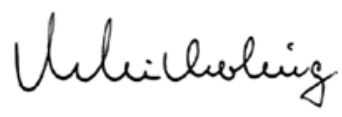

Michael Sticherling 